\title{
Cardiovascular autonomic control in patients undergoing left ventricular assist device (LVAD) support and pharmacologic therapy
}

\author{
David Nunan $^{\mathrm{a}, *}$, Gavin R.H. Sandercock ${ }^{\mathrm{b}}$, Robert S. George ${ }^{\mathrm{c}, \mathrm{d}}$, Djordje G. Jakovljevic ${ }^{\mathrm{e}}$, Gay Donovan ${ }^{\mathrm{f}}$, \\ Robert Bougard ${ }^{\text {c }}$, Magdi H. Yacoub ${ }^{\text {d }}$, David A. Brodie ${ }^{\text {f }}$, Emma J. Birks d,g \\ a Department of Primary Care Health Sciences, University of Oxford, Oxford, UK \\ ${ }^{b}$ Centre for Sports and Exercise Science, University of Essex, Colchester, UK \\ ${ }^{c}$ Royal Brompton and Harefield NHS Foundation Trust, Harefield, Middlesex, UK \\ ${ }^{d}$ Heart Science Centre, Imperial College, Harefield, Middlesex, UK \\ e Institute for Ageing and Health, Newcastle University, England, UK \\ ${ }^{\mathrm{f}}$ Research Centre for Society and Health, Buckinghamshire New University, High Wycombe, UK \\ ${ }^{\mathrm{g}}$ University of Louisville, Louisville, KY, USA
}

\section{A R T I C L E I N F O}

Article history:

Received 15 October 2012

Accepted 8 July 2013

Available online $\mathrm{xxxx}$

\section{Keywords:}

Bridge-to-recovery

Reverse remodelling

Heart rate variability

Normalisation

Prognosis

\begin{abstract}
A B S T R A C T
Objectives: The objective of the study is to determine cardiac autonomic control in patients undergoing assessment for and/or LVAD therapy.

Methods: Heart rate variability (HRV) was measured in 17 explanted LVAD, 17 implanted LVAD and 23 NYHA III-IV classified chronic heart failure (CHF) patients and ten healthy matched controls under three conditions: supine free breathing, standing and supine controlled breathing. Five measures of HRV were assessed: mean R-R interval (mR-R), high frequency (HF) and low frequency (LF) spectral power, LF in normalised units (LFnu), and LF to HF (LF:HF) ratio.

Results: Repeat measures ANOVA showed significant $(\mathrm{p}<0.05)$ differences in HRV between all three conditions within groups. Lower values were observed in $\mathrm{CHF}$ for $\mathrm{LF}$ (in log natural units) compared with explanted patients $(-1.4$ [95\% $\mathrm{Cl}-2.6$ to -0.7$], \mathrm{p}=0.04)$ and controls $(-2.1[-3.5$ to -0.7$], \mathrm{p}=0.001)$ and for LF: HF compared with implanted patients under paced breathing conditions $(z=-2.7, \mathrm{p}=0.007)$ and controls in standing $(z=-2.9, \mathrm{p}=0.004)$ and paced breathing conditions $(z=-2.3, \mathrm{p}=0.02)$. However, no significant differences were seen between explanted, implanted and control groups under any condition.

Conclusions: Patients implanted with an LVAD and explanted from a LVAD following myocardial recovery demonstrate a more normal dynamic response to autonomic stimuli and have a lower HRV risk profile compared to CHF patients.
\end{abstract}

(c) 2013 Elsevier Ireland Ltd. All rights reserved.

\section{Introduction}

Chronic heart failure (CHF) is a complex, multifactorial syndrome characterised by: inadequate tissue perfusion, fluid retention, skeletal muscle abnormalities, progressive activation of the neuroendocrine system (e.g. RAAS) and inappropriate immune response [1]. CHF patients demonstrate severe derangement of the autonomic nervous system (ANS) [2,3] characterised by chronic sympathetic hyperactivation [2], vagal withdrawal [3], resetting of the baroreflex sensitivity [4] and decreased peripheral and central responsiveness to adrenergic input [5].

This chronic sympathetic activation manifests as greatly altered heart rate variability (HRV). Overall R-R interval variation is lower in CHF patients who die compared with survivors [6-8]. Spectral

\footnotetext{
* Corresponding author at: Department of Primary Care Health Sciences, University of Oxford, 23-38 Hythe Bridge Street, Oxford, OX1 2ET, UK. Tel.: +44 1865 617938; fax: + 441865289287 .

E-mail address: david.nunan@phc.ox.ac.uk (D. Nunan).
}

analysis of HRV produces a characteristic HRV profile in CHF with attenuated variability both in the low frequency (LF, 0.04-0.15 Hz) and reduced low-to-high frequency ratio (LF:HF) which both predict patient mortality $[9,10]$.

Historically, the only cure for end stage CHF has been transplantation. In post-transplant patients, there is some restoration in autonomic modulation of the native sinus node. While there is also some evidence for partial (sympathetic) reinnervation of the donor heart, autonomic control and HRV remain well below normal levels [11].

Using left ventricular assisting devices (LVADs) to bridge patients to transplantation, destination therapy [12], or recovery [13] has become successful practise [14]. In combination with optimal pharmacotherapy, our group has been able to reverse many of the pathological changes associated with end stage heart failure and up to two thirds of patients with dilated cardiomyopathy can be explanted [15]. These patients may avoid transplantation and tend to lead higher quality lives than transplant patients [16]. 
Sudden cardiac death (SCD) remains the most likely mode of death for CHF patients [17] due to autonomic dysfunction and poor innervations respectively. While cardiac function and exercise capacity [18] of explanted LVAD patients exceed that of CHF sufferers, it remains unknown whether the same is true for cardiac autonomic function.

The aim of the present study was to assess the impact of 'bridging to recovery' on autonomic function in patients diagnosed with end stage heart failure undergoing assessment for and/or LVAD support therapy.

\section{Methods}

\subsection{Patients}

Heart rate variability was assessed in an opportunity sample of 17 explanted LVAD patients (age $36 \pm 11$ years, 12 men), 17 currently implanted patients ( $33 \pm 10$ years, 16 men), 23 NHYA III-IV classified chronic heart failure (CHF) patients ( $42 \pm 12$ years, 13 men) and ten healthy, age- and sex-matched controls from hospital and university staff ( $37 \pm 12$ years, 8 men). Assessments took place over the period of January 2004 to July 2008, therefore LVAD previously implanted in the explanted patients consisted of: a HeartMate $1(n=8)$; a HeartMate $2(n=5)$ (Thoratec Corporation, Pleasanton, CA, USA) a Levitronix $(n=1)$ (Thoratec Corporation, Pleasanton, CA, USA), a Heartware $(\mathrm{n}=1)$ ) (Heartware, Miami, USA) or a Jarvik 2000 (Jarvik Heart Inc. NY, USA, $\mathrm{n}=2$ ). All patients had been implanted only once but one had received additional right ventricular assistance from a Thoratec VAD (Thoratec Corporation, Pleasanton, CA, USA). In twelve explanted patients, the original diagnosis was idiopathic dilated cardiomyopathy (IDCM), in four it was peripartum cardiomyopathy and one had myocarditis.

Currently implanted LVAD patients had: a HeartMate $1(n=4)$; a HeartMate 2 $(n=7)$; a Heartware $(n=1)$; a Jarvik $2000(n=3)$, or a Thoratec $(n=2)$. Twelve patients had IDCM, one peripartum cardiomyopathy, three ischaemic heart disease (IHD), and one had myocarditis.

Of the CHF patients, 11 were diagnosed with IDCM, four with peripartum cardiomyopathy, six with IHD and two had myocarditis. Further anthropometric, physiological and pharmacological data are displayed in Table 1.

The control group was matched for age and sex with the explanted LVAD patient group. All controls were either sedentary or moderately physically active. None had a history of cardioneuroregulatory disorders. None were taking any prescribed medications, with the exception of oral contraceptives, at the time of testing. Due to the oligomenorrhoaeic nature of the female CHF patients, menstrual cycle phase was not controlled for in this group or their matched controls.

The local NHS ethics committee approved the research protocol and informed consent was obtained from all participants. The investigation conforms with the principles outlined in the Declaration of Helsinki.

\subsection{Instrumentation}

Two lead ECGs were recorded using a TF5 heart rate variability analysis system (Advanced Medical Diagnostics Ltd., Leeds, UK). This is a commercially available unit, which is mobile as well as being relatively simple and quick to fit to patients. The hardware and software components of the TF5 and its comparative validity with a 12 lead ECGs have been described previously [19]. The TF5 HRV analysis software was used to analyse the R-R interval data via fast Fourier transformation. All recording and analysis protocols surpass the criteria for sampling rates, conversion rates and mathematical treatment of data for HRV analysis [20].

\subsection{Protocol}

After fitting the patients with the TF5 chest strap in accordance with manufacturers' instructions, patients were allowed resting supine for 5-min. After this time, 5-min ECGs were recorded in three conditions: supine with free breathing, standing and supine with controlled breathing at 12 breaths $\cdot \min ^{-1}$. The supine condition was used as a baseline measure of resting autonomic modulation. The standing condition was used to represent orthostatic challenge, known to evoke sympathetic responses in healthy subjects. Finally the controlled breathing condition was used to stimulate vagal modulation of the SA node. We have reported the validity and reliability of this testing protocol in healthy participants previously [19]. All R-R interval data were first filtered using the automated algorithms available in the software. Additional manual ECG analysis, non-sinus beat rejection and consequent interpolation were also performed by a highly experienced HRV researcher.

All HRV measures recommended for use during short-term data collection were calculated [20]. In the frequency domain: high frequency spectral power (HF, 0.15$0.40 \mathrm{~Hz}$ ), low frequency spectral power (LF $0.04-0.15 \mathrm{~Hz}$ ) normalised low and high frequency spectral power (LFnu, HFnu), and the ratio of low to high frequency spectral power (LF:HF). In the time domain: mean R-R interval, standard deviation or normal-to-normal intervals (SDNN) and the root mean square of successive interval differences (rMSSD) were measured.

\subsection{Statistical analysis}

Two-way mixed (group by position) analysis of variance was used to determine whether differences between conditions (i.e. position and breathing) and between control, CHF, implanted and explanted patients (group) existed. Analysis was carried out on HRV measures representative of vagal modulation (HF), mixed sympathovagal and baroreflex activity (LF) and its normalised units (LFnu), sympathovagal interaction (LF:HF) and mean R-R interval. Due to heterogeneous variances and non-normal distributions which could not be modified successfully by transformation, LF:HF was analysed using repeated nonparametric Kruskal-Wallis tests $(n=3)$ with post-hoc Mann-Whitney U tests. For all tests, a two-sided value of $\mathrm{p}<0.05$ was considered statistically significant. We present data as mean \pm SD and the $95 \%$ confidence interval/limits $(\mathrm{CI})$ for the mean to demonstrate the magnitude of effects across each of the groups.

Table 1

Descriptive characteristics of implanted and explanted LVAD patients.

\begin{tabular}{|c|c|c|c|c|}
\hline & $\mathrm{CHF}$ & Implanted & Explanted & Control \\
\hline $\mathrm{N}$ & 23 & 17 & 17 & 10 \\
\hline Males/females & $13 / 10$ & $16 / 1$ & $12 / 5$ & $8 / 2$ \\
\hline Age (years) & $42.3 \pm 12.1$ & $32.8 \pm 10.2$ & $36.2 \pm 10.9$ & $37.0 \pm 12.2$ \\
\hline Mass $(\mathrm{kg})$ & $82.1 \pm 19.5$ & $78.7 \pm 19.4$ & $86.7 \pm 24.9$ & $87.2 \pm 13.0$ \\
\hline Stature $(\mathrm{cm})$ & $168.6 \pm 11.4$ & $180.4 \pm 8.5$ & $176.1 \pm 12.0$ & $178.2 \pm 11.4$ \\
\hline BMI $\left(\mathrm{m}^{2} \cdot \mathrm{kg}\right)$ & $29.0 \pm 7.7$ & $24.0 \pm 4.8$ & $27.6 \pm 5.7$ & $27.3 \pm 1.7$ \\
\hline $\operatorname{BSA}\left(\mathrm{m}^{2}\right)$ & $1.93 \pm 0.48$ & $1.98 \pm 0.55$ & $2.18 \pm 0.70$ & $2.17 \pm 0.43$ \\
\hline Time from explant/implant/diagnosis (days) & $1291 \pm 1577$ & $184 \pm 136$ & $718 \pm 639$ & $\mathrm{n} / \mathrm{a}$ \\
\hline NYHA class & $3.3 \pm 0.7$ & $1.8 \pm 0.7$ & $1.1 \pm 0.3$ & $\mathrm{n} / \mathrm{a}$ \\
\hline LVEF at time of test (\%) & $39.5 \pm 14.2$ & $47.8 \pm 9.4$ & $62.6 \pm 13.3$ & $\mathrm{n} / \mathrm{a}$ \\
\hline \multicolumn{5}{|l|}{ Aetiology (number) } \\
\hline IDCM & 11 & 12 & 12 & $\mathrm{n} / \mathrm{a}$ \\
\hline IHD & 5 & 3 & 0 & $\mathrm{n} / \mathrm{a}$ \\
\hline MYO & 3 & 1 & 1 & $\mathrm{n} / \mathrm{a}$ \\
\hline $\mathrm{PP}$ & 4 & 1 & 4 & $\mathrm{n} / \mathrm{a}$ \\
\hline Clenbuterol treatment (\%) & 0 & 25 & 100 & $\mathrm{n} / \mathrm{a}$ \\
\hline Clenbuterol at time of test (\%) & 0 & 6 & 0 & $\mathrm{n} / \mathrm{a}$ \\
\hline Digitalis (\%) & 27 & 38 & 100 & $\mathrm{n} / \mathrm{a}$ \\
\hline$\beta$-Blockers (\%) & 64 & 87 & 87 & $\mathrm{n} / \mathrm{a}$ \\
\hline ACE-inhibitors (\%) & 27 & 62 & 100 & $\mathrm{n} / \mathrm{a}$ \\
\hline ARB $(\%)$ & 58 & 62 & 87 & $\mathrm{n} / \mathrm{a}$ \\
\hline Spirolactolone (\%) & 41 & 50 & 62 & $\mathrm{n} / \mathrm{a}$ \\
\hline Other (\%) & 60 & 87 & 38 & $\mathrm{n} / \mathrm{a}$ \\
\hline
\end{tabular}

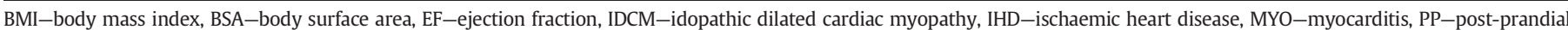

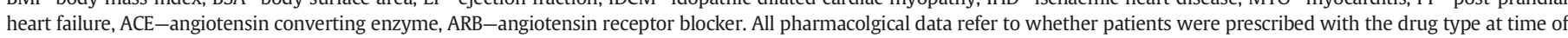
HRV measurement except clenbuterol treatment - refers to whether the patient had ever received clenbuterol; n/a-non-applicable. 
Further non-statistical comparisons were made by comparing mean $( \pm$ SD) levels of significant short-term HRV risk factors reported previously $[9,10]$ with values obtained for explanted, implanted and CHF patients and for comparison, control subjects. This analysis allowed visual evaluation of spectral HRV profiles at different points during 'bridging to recovery' in comparison with heart failure patients and healthy control individuals.

\section{Results}

Table 1 provides the descriptive characteristics of the implanted, explanted and CHF patient groups. Implanted and explanted patients were similar in terms of age and anthropometric measures, disease aetiology, and geometric echocardiographic measures. The CHF patients were older and some differences in pharmacotherapy existed between groups, particularly in the frequency of use of clenbuterol.

\subsection{Comparisons between patient groups and healthy controls}

Table 2 shows the changes in R-R interval, HF power as natura logarithm $\left(\operatorname{ln~} \mathrm{ms}^{2}\right), \mathrm{LF}(\ln )$ and LF:HF under supine, standing and supine controlled breathing conditions in implanted, explanted and CHF patients and healthy controls.

Repeated measures ANOVA showed significant $(\mathrm{p}<0.05)$ differences for all three conditions within groups. There were significant group differences across conditions for LF in $\log$ transformed $(\mathrm{LF}(\ln ), \mathrm{p}=0.02)$ and normalised units (LFnu, $\mathrm{p}=0.01$ ) and for LF:HF ( $p=0.007$ ) in the paced breathing condition only. There were no significant between group differences in $\mathrm{R}-\mathrm{R}$ interval $(p=0.08)$ or $H F(\ln )(p=0.13)$. Post-hoc analyses demonstrated that nearly all group differences were between the CHF patients and one or more of the remaining three groups as follows: significantly lower values for $\operatorname{LF}(\ln )$ compared with explanted patients $(-1.4[95 \% \mathrm{CI}-2.6$ to -0.7$], \mathrm{p}=0.04)$ and controls $(-2.1[-3.5$ to -0.7$], \mathrm{p}=0.001)$; for LFnu compared with implanted $(-19.4$ $[-38.1$ to -0.5$], \mathrm{p}=0.04)$ and controls $(-20.3[-38.6$ to -1.9$]$, $\mathrm{p}=0.03)$; and for LF:HF compared with implanted patients under paced breathing conditions $(z=-2.7, \mathrm{p}=0.007)$ and controls in standing $(z=-2.9, \mathrm{p}=0.004)$ and paced breathing conditions $(z=-2.3, \mathrm{p}=0.02)$.

Due to the relatively small number of patients available, a visual analysis of changes in LF:HF ratio due to standing and controlled breathing was also performed. Fig. 1 shows the expected increase in LF:HF due to standing compared with values measured in the supine position in control, heart failure and implanted and explanted patients. The pattern of change was similar in both implanted and explanted patients and their values were more closely matched to those of the control group compared to those of CHF patients.

\section{Discussion}

The aim of this study was to examine the potential of LVAD unloading and optimal pharmacotherapy to alter cardiac autonomic nervous system activity in end-stage heart failure patients, a condition typically characterised by severe autonomic derangement. In explanted patients, our power-spectral analysis of HRV demonstrated patterns of cardiac ANS activity that were similar to those of healthy, age-matched controls.

Successful explantation is the desired clinical endpoint for LVAD patients who are 'bridged to recovery' $[13,14]$ and although not all patients achieve this some degree of reverse modelling occurs in most [21]. The present data show that in currently implanted LVAD patients, there appears to be a partial normalisation of the HRV profile as we found no difference between controls and implanted patients for mean R-R interval or spectral HRV values.

\subsection{Low frequency spectral power: explants and implants}

The characteristic HRV profile of heart failure patients shows severely attenuated overall variability, particularly in the low frequency spectral component ( $\mathrm{LF}, 0.04-0.15 \mathrm{~Hz}$ ). Values for LF predictive of mortality range from $3.3 \mathrm{ln} \mathrm{ms} \mathrm{s}^{2}$ from Holter recordings [22] to between $<13 \mathrm{~ms}^{2}$ [9] and $20 \mathrm{~ms}^{2}$ [10] when extracted from more-stationary recordings as in the present study. We have previously calculated estimated normal values for the LF frequency component to be around $519 \pm 219 \mathrm{~ms}^{2}$ or $5.0 \pm 1.8 \mathrm{ln} \mathrm{ms}^{2}$ [23]. HRV measures should be compared only between comparable R-R interval recording conditions and the following discussion relates to data collected supine with free breathing.

Mean LF power in our controls was within the normal range. Remarkably however, and despite having been previously diagnosed with end-stage heart failure, mean LF values of explanted patients were similar to that of controls and therefore population norms. Perhaps more importantly, both mean and the lower 95\% CI (4.7 [3.7 to 5.8]) values were well in excess of values known to predict mortality in CHF. While we do not have longitudinal data to describe the changes in HRV from pre-implantation to explantation, it is clear that LF power

Table 2

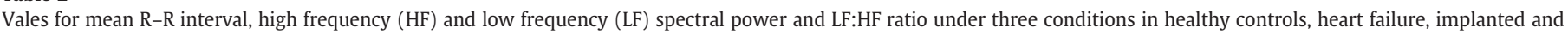
explanted LVAD patients.

\begin{tabular}{|c|c|c|c|c|}
\hline & Heart failure & Implanted & Explanted & Control \\
\hline \multicolumn{5}{|c|}{ Measures obtained supine } \\
\hline $\mathrm{LF}(\ln )$ & $4.2 \pm 1.7(3.0$ to 5.4$)$ & $5.0 \pm 1.4(3.9$ to 6.0$)$ & $5.6 \pm 1.5(4.6$ to 6.5$)$ & $6.3 \pm 1.6(5.3$ to 7.2$)$ \\
\hline $\mathrm{HF}(\ln )$ & $4.1 \pm 2.1(2.7$ to 5.5$)$ & $4.2 \pm 1.4(3.1$ to 5.3$)$ & $5.4 \pm 0.9(4.8$ to 5.9$)$ & $5.5 \pm 1.3(4.6$ to 6.4$)$ \\
\hline $\mathrm{LF}(\mathrm{nu})$ & $52 \pm 23(37$ to 68$)$ & $68 \pm 12(59$ to 77$)$ & $53 \pm 26(38$ to 69$)$ & $66 \pm 17(53$ to 78$)$ \\
\hline LF:HF & $1.8 \pm 1.9(0.6$ to 3.1$)$ & $3.2 \pm 3.2(0.6$ to 5.5$)$ & $2.6 \pm 3.5(0.4$ to 4.9$)$ & $3.4 \pm 3.6(0.6$ to 6.0$)$ \\
\hline \multicolumn{5}{|c|}{ Measures obtained standing } \\
\hline $\mathrm{mR}-\mathrm{R}(\mathrm{ms})$ & $759 \pm 145(662$ to 857$)$ & $694 \pm 82(631$ to 757$)$ & $766 \pm 78(719$ to 813$)$ & $795 \pm 81(737$ to 853$)$ \\
\hline $\operatorname{LF}(\ln )$ & $3.8 \pm 1.1(3.1$ to 4.6$)$ & $4.8 \pm 1.4(3.7$ to 5.8$)$ & $4.7 \pm 1.2(4.0$ to 5.4$)$ & $6.0 \pm 1.2(5.2$ to 6.9$)$ \\
\hline $\mathrm{HF}(\ln )$ & $3.5 \pm 1.9(2.3$ to 4.8$)$ & $3.7 \pm 1.5(2.6$ to 4.9$)$ & $3.7 \pm 1.1(3.0$ to 4.3$)$ & $4.2 \pm 1.4(3.2$ to 5.2$)$ \\
\hline $\mathrm{LF}(\mathrm{nu})$ & $56 \pm 25(39$ to 73$)$ & $74 \pm 20(58$ to 89$)$ & $74 \pm 17(64$ to 84$)$ & $85 \pm 5(81$ to 89$)$ \\
\hline LF:HF & $2.5 \pm 2.7(0.7$ to 4.3$)$ & $5.3 \pm 5.2(1.3$ to 9.3$)$ & $4.9 \pm 4.9(1.6$ to 7.8$)$ & $7.0 \pm 5.6(3.1$ to 11.4$)$ \\
\hline \multicolumn{5}{|c|}{ Measures obtained supine with controlled breathing } \\
\hline $\mathrm{mR}-\mathrm{R}(\mathrm{ms})$ & $858 \pm 210(717$ to 999$)$ & $770 \pm 121(677$ to 862$)$ & $890 \pm 97(832$ to 949$)$ & $978 \pm 113(898$ to 1059$)$ \\
\hline $\operatorname{LF}(\ln )$ & $3.6 \pm 1.6(2.5$ to 4.7$)$ & $4.8 \pm 1.2(3.9$ to 5.7$)$ & $5.4 \pm 1.5(4.5$ to 6.3$)$ & $5.6 \pm 1.3(4.7$ to 6.5$)$ \\
\hline $\mathrm{HF}(\ln )$ & $4.5 \pm 2.4(2.9$ to 6.1$)$ & $4.6 \pm 1.5(3.4$ to 5.7$)$ & $6.1 \pm 1.4(5.4$ to 7.0$)$ & $5.6 \pm 1.2(4.7$ to 6.5$)$ \\
\hline $\mathrm{LF}(\mathrm{nu})$ & $32 \pm 25(15$ to 49$)$ & $56 \pm 21(40$ to 72$)$ & $35 \pm 24(21$ to 50$)$ & $50 \pm 23(33$ to 66$)$ \\
\hline LF:HF & $0.7 \pm 1.1(0.0$ to 1.9$)$ & $1.4 \pm 1.2(0.8$ to 2.9$)$ & $0.8 \pm 0.9(0.3$ to 1.4$)$ & $1.6 \pm 1.5(0.4$ to 2.7$)$ \\
\hline
\end{tabular}

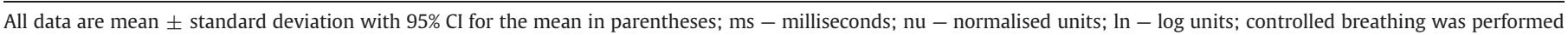
at12 breaths $\bullet \mathrm{min}^{-1}$. 


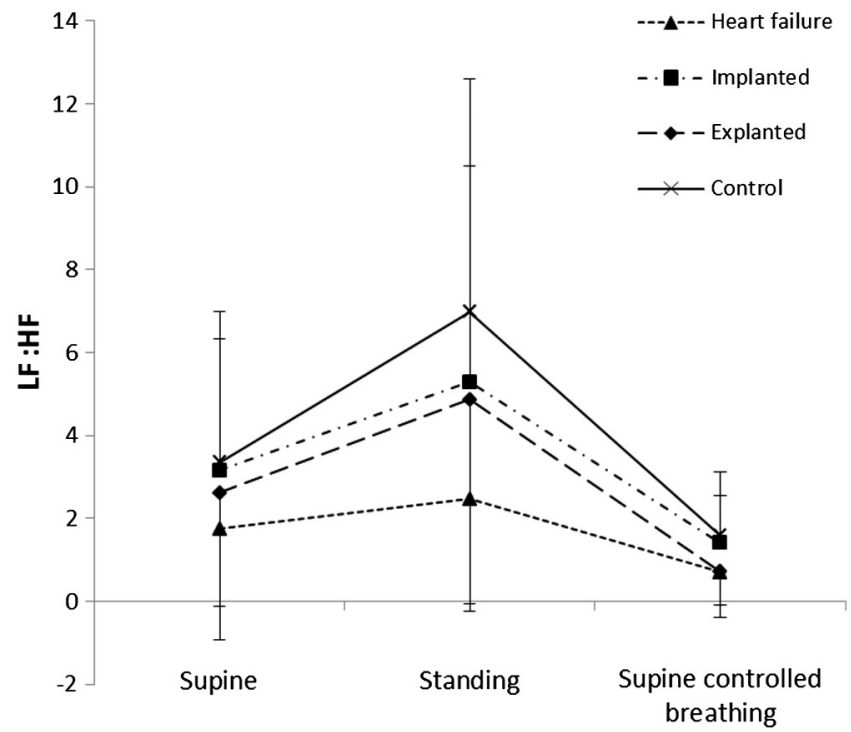

Fig. 1. LF:HF ratio values in LVAD implanted, LVAD explanted and chronic heart failure patients and controls.

of both explanted and currently implanted patients is much more similar to normal values than to the heart failure patients measured here. Explanted and currently implanted LVAD patients therefore demonstrate a lower HRV risk profile compared with chronic heart failure patients. If we assume that implanted and explanted patients' LF values were similar to those of the heart failure group prior to LVAD therapy, their current, near-normal levels demonstrate the potential for autonomic recovery following our treatment programme.

\subsection{Low to high frequency ratio: explants and implants}

We analysed the ratio of low to high frequency powers (LF:HF) ratio to evaluate the interaction between both ANS branches. It should, however, be noted that the conditions under which the behaviour of LF:HF actually matche sympathetic activation and/or vagal withdrawal are somewhat limited [24]. Interpretation of LF:HF in patients demonstrating severe autonomic derangement is particularly problematic $[2,3]$. Whereas in healthy populations LF:HF usually reflects an increase in sympathetic outflow (and/or vagal withdrawal) values in LF:HF and in CHF patients with excessive sympathetic outflow is, paradoxically, very low. This is because of very low values for LF due to severely attenuated baroreflex sensitivity in these patients [25]. Regardless of its physiological meaning, low LF:HF is a risk factor of mortality and cardiac event in CHF patients [9,10]. The reduced LF component in the HRV profile of CHF patients results in a low LF:HF ratio and the most sensitive values to predict mortality reported by La Rovere et al. were $<0.37$ and $<0.43$ [9].

In the present study, values for LF:HF were much higher and almost identical in explants, implants and controls. LF:HF showed great intra-individual variation and while the lower 95\% CI in explants was below the cut-point of La Rovere et al. [9], this was not the case in implanted patients and the mean values in both cases do not suggest that these patients are at significantly heightened risk of SCD [10]. From these data, we propose that the balance between LF and HF power is likely to be partially restored in CHF patients during LVAD support and that this restoration of balance is maintained post-explantation.

\subsection{Autonomic response to standing and controlled breathing}

The response of HRV measures to orthostatic challenge and deep breathing has not been used in prognosis in CHF patients, however it remains a useful tool by which to assess dynamic control of ANS. Typically, this is deranged in CHF patients and we aimed to determine whether a normal profile of change in HRV measures had returned in LVAD patients. Standing comprises an orthostatic challenge, the autonomic response to which is typified by a reproducible decreased HF and increased LF contribution to total spectral power [26]. The use of controlled breathing typically increases the vagal contribution to SA node control [27]. CHF patients with sympathetic hyperactivity tend to show blunted decreases in vagal indices (HF, HFnu) and increases in indices (LFnu) of sympathetic activation [26]. While this was the case in our CHF patients, the LF:HF for implanted and explanted patients displayed a typical response to standing (increase) and decreased under the increased vagal outflow created by controlled breathing (Fig. 1). Even the magnitudes of these changes were highly similar between groups the increase in LF:HF upon standing demonstrates combined vagal withdrawal and sympathetic activation (increased relative LF contribution) mediated via the baroreflex. These are the first data therefore, to demonstrate a normalisation of HRV indices recorded under resting conditions that indicate a reduced likelihood of cardiac events in currently implanted and explanted LVAD recipients. These data are also the first to demonstrate normality of the dynamic autonomic responses to simple sympathetic and vagal perturbations.

\subsection{Potential mechanisms of recovery}

The unloading of the failing myocardium by LVAD implantation, combined with optimal pharmacotherapeutic interventions results in multiple favourable physiological changes including partial restoration of hemodynamic responses to exercise[18], 'reverse remodelling'[28] of the myocardium which has been well described at both macrostructural [29] and cellular levels [30]. Burkhoff et al. [29] suggest restoration of the 'normal neurohormonal milieu' is a precursor to such adaptations but descriptions of this autonomic normalisation are scarce. Scintigraphy demonstrates that hemodynamic improvements are accompanied by improved sympathetic innervation of failing myocardium in implanted patients [31]. Changes in sympathetic innervation correlate improvements in clinical measures including B-type natriuretic peptide and pulmonary systolic pressure [32]. LVAD implantation may increase myocardial noradrenalin via the renin-angiotensin-aldosterone pathway [30] but the optimised pharmacotherapy [15] in these patients reduces or negates this change. No group has yet published data describing changes in integrated cANS function (sympathovagal interaction) associated with LVAD therapy.

In addition to being a significant prognostic marker, HRV provide at least some information regarding the overall functioning of the cANS. The near-normal resting HRV index values observed in implanted and explanted patients suggest a degree of autonomic normalisation. It appears that by unloading the left ventricle, the cycle of sympathetic over activity is corrected to a significant degree. These data show that LVAD therapy potentially restores predictable responses and near normal values for reflex cardiac autonomic activity in the cANS of what were once most-likely severely deranged systems of patients with end stage heart failure.

\section{Limitations}

We acknowledge that the major limitation of this study is the cross-sectional design. During the study period, only one patient with end-stage heart failure was implanted then explanted from LVAD. Moreover, only two patients moved from implanted to explanted states. The duration of a study required to collect longitudinal data would appear to be more than several years. Of note is that the thirty-seven patients in the present study represent a very large proportion of survivors of LVAD implantation (and explantation) over a period of approximately five years. In fact, they represented the entire ongoing LVAD implant population from our hospital that at the time of the study were in sinus rhythm and/or not paced. In turn, they also 
represent a very large proportion of the total UK LVAD population. Only three patients were not able to have HRV measured. In one case this was due to persistent atrial fibrillation and two other cases due to the use of implantable pacing devices.

We recommended that a longitudinal study, tracking changes in HRV and possibly baroreflex gain or other sympathetic measurement be carried out to determine if LVAD therapy can lead to definite autonomic recovery in successfully treated patients. A single-centre prospective study may be prohibitively long or may have very small subject numbers. Such data collection would no doubt be made easier by collaboration and co-operation between units in order to combine their experience and data to provide a better overall understanding of the outcomes from this rare but seemingly very successful intervention.

\section{Conclusions}

Patients implanted with and explanted from a LVAD following myocardial recovery demonstrate a lower HRV risk profile compared to patients with end-stage heart failure and have a dynamic response to autonomic stimuli similar to healthy matched controls.

\section{Acknowledgements}

The authors thank the participants who took part in this research, and we thank the members of the Cardiology Department at Harefield Hospital for their cooperation and assistance during patient assessments.

The authors of this manuscript have certified that they comply with the Principles of Ethical Publishing in the International Journal of Cardiology.

Funding

This research received no specific grant from any funding agency in the public, commercial or not-for-profit sectors.

\section{References}

[1] Adamopoulos S, Parissis JT, Kremastinos DT. New aspects for the role of physical training in the management of patients with chronic heart failure. Int J Cardiol 2003;90:1-14.

[2] Leimbach Jr WN, Wallin BG, Victor RG, et al. Direct evidence from intraneural recordings for increased central sympathetic outflow in patients with heart failure. Circulation 1986;73:913-9.

[3] Nolan J, Flapan AD, Capewell S, et al. Decreased cardiac parasympathetic activity in chronic heart failure and its relation to left ventricular function. Br Heart J 1992;67:482-5.

[4] Mortara A, La Rovere MT, Pinna GD, et al. Arterial baroreflex modulation of heart rate in chronic heart failure: clinical and hemodynamic correlates and prognostic implications. Circulation 1997;96:3450-8.

[5] Bristow MR, Ginsburg R, Minobe W, et al. Decreased catecholamine sensitivity and beta-adrenergic-receptor density in failing human hearts. N Engl J Med 1982;307: 205-11.

[6] Nolan J, Batin PD, Andrews R, et al. Prospective study of heart rate variability and mortality in chronic heart failure: results of the United Kingdom heart failure evaluation and assessment of risk trial (UK-heart). Circulation 1998;98:1510-6.

[7] Ponikowski P, Anker SD, Chua TP, et al. Depressed heart rate variability as an independent predictor of death in chronic congestive heart failure secondary to ischemic or idiopathic dilated cardiomyopathy. Am J Cardiol 1997;79:1645-50.

[8] Yi G, Goldman JH, Keeling PJ, et al. Heart rate variability in idiopathic dilated cardiomyopathy: relation to disease severity and prognosis. Heart 1997;77:108-14.
9] La Rovere MT, Pinna GD, Maestri R, et al. Short-term heart rate variability strongly predicts sudden cardiac death in chronic heart failure patients. Circulation 2003;107:565-70

[10] Lucreziotti S, Gavazzi A, Scelsi L, et al. Five-minute recording of heart rate variability in severe chronic heart failure: correlates with right ventricular function and prognostic implications. Am Heart J 2000;139:1088-95.

[11] Lord SW, Senior RR, Das M, et al. Low-frequency heart rate variability: reproducibility in cardiac transplant recipients and normal subjects. Clin Sci (Lond) 2001;100:43-6.

[12] Slaughter MS, Rogers JG, Milano CA, et al. Advanced heart failure treated with continuous-flow left ventricular assist device. N Engl J Med 2009;361: 2241-51.

[13] Yacoub MH. A novel strategy to maximize the efficacy of left ventricular assist devices as a bridge to recovery. Eur Heart J 2001;22:534-40.

[14] Reinlib L, Abraham W. Recovery from heart failure with circulatory assist: a working group of the National, Heart, Lung, and Blood Institute. J Card Fail 2003;9: 459-63.

[15] Birks EJ, Tansley PD, Hardy J, et al. Left ventricular assist device and drug therapy for the reversal of heart failure. N Engl J Med 2006;355:1873-84.

[16] George RS, Yacoub MH, Bowles CT, et al. Quality of life after removal of left ventricular assist device for myocardial recovery. J Heart Lung Transplant 2008;27: 165-72.

[17] Sandercock GR, Brodie DA. The role of heart rate variability in prognosis for different modes of death in chronic heart failure. Pacing Clin Electrophysiol 2006;29: 892-904.

[18] Jakovljevic DG, George RS, Donovan G, et al. Comparison of cardiac power output and exercise performance in patients with left ventricular assist devices, explanted (recovered) patients, and those with moderate to severe heart failure. Am J Cardiol 2010;105:1780-5.

[19] Sandercock GR, Bromley P, Brodie DA. Reliability of three commercially available heart rate variability instruments using short-term (5-min) recordings. Clin Physiol Funct Imaging 2004;24:359-67.

[20] TaskForce. Heart rate variability. Standards of measurement, physiological interpretation, and clinical use. Task Force of the European Society of Cardiology and the North American Society of Pacing and Electrophysiology. Eur Heart J 1996; 17:354-81.

[21] Birks EJ, Yacoub MH, Banner NR, Khaghani A. The role of bridge to transplantation: should LVAD patients be transplanted? Curr Opin Cardiol 2004;19:148-53.

[22] Galinier M, Pathak A, Fourcade J, et al. Depressed low frequency power of heart rate variability as an independent predictor of sudden death in chronic heart failure. Eur Heart J 2000;21:475-82.

[23] Nunan D, Sandercock GR, Brodie DA. A quantitative systematic review of normal values for short-term heart rate variability in healthy adults. Pacing Clin Electrophysiol 2010;33:1407-17.

[24] Eckberg DL. Sympathovagal balance: a critical appraisal. Circulation 1997;96: 3224-32.

[25] Osterziel KJ, Hanlein D, Willenbrock R, et al. Baroreflex sensitivity and cardiovascular mortality in patients with mild to moderate heart failure. Br Heart J 1995;73:517-22.

[26] Malfatto G, Branzi G, Gritti S, et al. Different baseline sympathovagal balance and cardiac autonomic responsiveness in ischemic and non-ischemic congestive heart failure. Eur J Heart Fail 2001;3:197-202.

[27] Sinnreich R, Kark JD, Friedlander Y, Sapoznikov D, Luria MH. Five minute recordings of heart rate variability for population studies: repeatability and age-sex characteristics. Heart 1998;80:156-62.

[28] Wohlschlaeger J, Schmitz KJ, Schmid C, et al. Reverse remodeling following insertion of left ventricular assist devices (LVAD): a review of the morphological and molecular changes. Cardiovasc Res 2005;68:376-86.

[29] Burkhoff D, Klotz S, Mancini DM. LVAD-induced reverse remodeling: basic and clinical implications for myocardial recovery. J Card Fail 2006;12:227-39.

[30] Klotz S, Burkhoff D, Garrelds IM, Boomsma F, Danser AH. The impact of left ventricular assist device-induced left ventricular unloading on the myocardial renin-angiotensin-aldosterone system: therapeutic consequences? Eur Heart J 2009;30:805-12.

[31] Miyagawa S, Sawa Y, Fukushima N, et al. Analysis of sympathetic nerve activity in end-stage cardiomyopathy patients receiving left ventricular support. J Heart Lung Transplant 2001;20:1181-7.

[32] Drakos SG, Athanasoulis T, Malliaras KG, et al. Myocardial sympathetic innervation and long-term left ventricular mechanical unloading. JACC Cardiovasc Imaging 2010;3:64-70. 